\title{
Experimental demonstration of a Hadamard gate for coherent state qubits
}

Tipsmark, Anders; Dong, Ruifang; Laghaout, Amine; Marek, Petr; Jezek, Miroslav; Andersen, Ulrik Lund

Published in:

Physical Review A

Link to article, DOI:

10.1103/PhysRevA.84.050301

Publication date:

2011

Document Version

Publisher's PDF, also known as Version of record

Link back to DTU Orbit

Citation $(A P A)$ :

Tipsmark, A., Dong, R., Laghaout, A., Marek, P., Jezek, M., \& Andersen, U. L. (2011). Experimental demonstration of a Hadamard gate for coherent state qubits. Physical Review A, 84(5), -.

https://doi.org/10.1103/PhysRevA.84.050301

\section{General rights}

Copyright and moral rights for the publications made accessible in the public portal are retained by the authors and/or other copyright owners and it is a condition of accessing publications that users recognise and abide by the legal requirements associated with these rights.

- Users may download and print one copy of any publication from the public portal for the purpose of private study or research.

- You may not further distribute the material or use it for any profit-making activity or commercial gain

- You may freely distribute the URL identifying the publication in the public portal 


\title{
Experimental demonstration of a Hadamard gate for coherent state qubits
}

\author{
Anders Tipsmark, ${ }^{1,}$ Ruifang Dong, ${ }^{2,1}$ Amine Laghaout, ${ }^{1}$ Petr Marek, ${ }^{3}$ Miroslav Ježek, ${ }^{3,1}$ and Ulrik L. Andersen ${ }^{1}$ \\ ${ }^{1}$ Department of Physics, Technical University of Denmark, Fysikvej, DK-2800 Kgs. Lyngby, Denmark \\ ${ }^{2}$ Quantum Frequency Standards Division, National Time Service Center (NTSC), Chinese Academy of Sciences, \\ 710600 Lintong, Shaanxi, China \\ ${ }^{3}$ Department of Optics, Palacký University, 17. listopadu 12, CZ-77146 Olomouc, Czech Republic
}

(Received 4 July 2011; published 8 November 2011)

\begin{abstract}
We discuss and make an experimental test of a probabilistic Hadamard gate for coherent state qubits. The scheme is based on linear optical components, nonclassical resources, and the joint projective action of a photon counter and a homodyne detector. We experimentally characterize the gate for the coherent states of the computational basis by full tomographic reconstruction of the transformed output states. Based on the parameters of the experiment, we simulate the fidelity for all coherent state qubits on the Bloch sphere.
\end{abstract}

DOI: 10.1103/PhysRevA.84.050301

PACS number(s): 03.67.Lx, 42.50.Ex

Measurement-based, linear optical quantum processors rely on offline prepared resources, linear optical transformations, and measurement-induced operations [1]. Among all measurement-based protocols, the most famous ones are the cluster state quantum processor where universal operations are executed by measuring a large entangled cluster state [2], and the linear quantum computer approach proposed by Knill, Laflamme, and Milburn [3]. The latter method is based on single-photon resources that interfere in a linear optical network and subsequently are measured to enforce the desired operation. Despite its seeming simplicity, the implementation of a fault tolerant operating algorithm is complex as it requires a very large overhead.

An alternative approach to measurement-based linear quantum computing has been put forward by Ralph et al. [4]. Rather than using discrete degrees of freedom (e.g., the polarization) of a single photon as the computational basis, it was suggested to use two mesoscopic coherent states $|\alpha\rangle$ and $|-\alpha\rangle$, where $\alpha$ is the amplitude. Although these states are only approximately orthogonal $(\langle\alpha \mid-\alpha\rangle \neq 0)$, resource-efficient and fault-tolerant quantum gates can be implemented: For a large coherent amplitude, that is, $\alpha>2$, deterministic gates can in principle be realized, although the experimental implementation is very challenging [5]. On the other hand, by employing a simpler physical implementation, nondeterministic gates can be realized for any value of $\alpha$, and for $\alpha>1.2$, the scheme was theoretically shown to be fault tolerant and resource efficient [6].

An even simpler implementation of a universal set of nondeterministic quantum gates was recently suggested by Marek and Fiurášek [7]. They proposed the physical realization of a single-mode and a two-mode phase gate as well as the Hadamard gate. In this Rapid Communication we present a proof of principle experiment of the probabilistic Hadamard gate for coherent state qubits. The implemented protocol is based on a squeezed state resource, linear operations as well as two projective measurements of discrete and continuous variable types. By injecting the computational basis states $(|\alpha\rangle$ and $|-\alpha\rangle)$ into the gate we partially characterize

*Corresponding author: anders.tipsmark@fysik.dtu.dk its function by reconstructing the Wigner functions of the transformed output states and calculate the fidelity with an ideally transformed state. Based on these results we perform a simulation of the gate performance for arbitrary coherent state qubits.

A Hadamard gate transforms the computational basis states $| \pm \alpha\rangle$ into the diagonal basis states $(|\alpha\rangle \pm|-\alpha\rangle) / \sqrt{N_{ \pm}}$, which we refer to as the even and odd coherent state qubits (CSQs) [8-15]. Such a transformation can be performed probabilistically using the circuit shown in Fig. 1(a). The gate is based on a supply of coherent state superposition resources which are assumed to have the same amplitude as the coherent states of the computational basis. The gate works by displacing the arbitrary CSQ input state $\left|\psi_{\text {in }}\right\rangle=(u|\alpha\rangle+v|-\alpha\rangle) / \sqrt{N}$, followed by a nondistinguishable subtraction of a single photon, from either the displaced input or the resource state. Physically, this can be done by reflecting a small part of either state using highly asymmetric beam splitters (ABS1,ABS2), interfering the resulting beams on a beam splitter (BS) with transmittivity $t$ and reflectivity $r$, and detecting one photon at the output with a single-photon detector. Theoretically this is described by the operator $r \hat{a}+t \hat{b}$, where $\hat{a}$ and $\hat{b}$ are annihilation operators corresponding to the subtraction of a photon from the displaced input and the coherent state superposition resource, respectively. As a final step the two-mode state is projected onto the single-mode quadrature eigenstate $|x\rangle$, where $x$ is the amplitude quadrature, by using a homodyne detector (HD). The resulting output state is

$$
u \frac{|\alpha\rangle+|-\alpha\rangle}{\sqrt{N_{+}}}+Y_{1}(u+v Z) \frac{|\alpha\rangle-|-\alpha\rangle}{\sqrt{N_{-}}},
$$

where

$$
Y_{1}=\frac{t}{2 r} \sqrt{\frac{N_{-}}{N_{+}}}, \quad Z=\frac{\langle x \mid 0\rangle}{\langle x \mid 2 \alpha\rangle} .
$$

By using a beam splitter (BS) with $t \ll r$ and setting the $x$ quadrature such that $Z \gg 1$ and $Z Y_{1}=1$, the Hadamard transform is implemented. The gate is probabilistic, and implemented by a hybrid detection system, using both discrete and continuous variable projections [16,17]. Its success is conditioned on the joint measurement of a photon and a quadrature measurement outcome with the value $x$. 
(a)
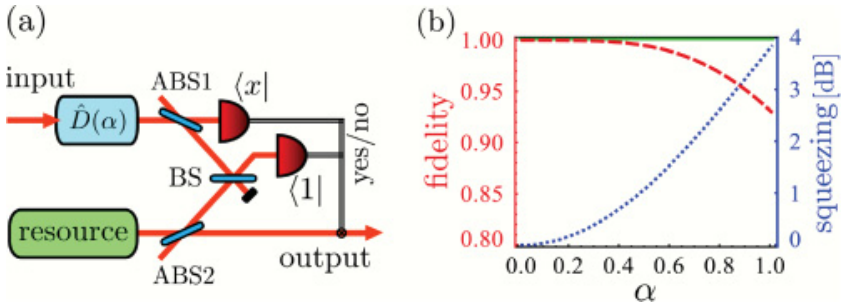

FIG. 1. (Color online) (a) Schematic of the Hadamard gate. The input coherent state qubit (CSQ) is displaced $(\hat{D})$ and mixed with a resource state at a beam splitter (BS). The output of the gate is conditioned by a single-photon detection $(\langle 1|)$ and a homodyne measurement $(\langle x|)$. (b) Gate fidelity as a function of the CSQ amplitude for an ideal coherent state superposition resource (solid green/light gray) and the squeezed state resource (dashed red/gray). The degree of squeezing that optimizes the fidelity is represented by the dotted blue/dark gray curve.

As an even coherent state superposition with small amplitude is reminiscent of a squeezed vacuum state, and this latter state is experimentally easier to prepare, we will in the following consider the replacement of the ideal resource with a squeezed vacuum state. With this substitution, the transformed state will have the following form:

$$
u \hat{S}(s)|0\rangle+Y_{2}(u+v Z) \hat{S}(s) \hat{a}^{\dagger}|0\rangle,
$$

where $s$ is the squeezing parameter which is related to the squeezing variance by $V=e^{-2 s}$, and the parameter $Y_{2}$ is now given by

$$
Y_{2}=-t \sinh (s) /(2 r \alpha) .
$$

Again, the requirement for optimal implementation of the Hadamard transform is $Z \gg 1$ and $Z Y_{2}=1$. Using this result we calculate the expected gate fidelity for various amplitudes $\alpha$ as shown by the dashed red/gray curve in Fig. 1(b). For the squeezed vacuum resource, we optimize the squeezing degree (shown by the dotted blue/dark gray curve) to obtain the highest fidelity which reaches unity for $\alpha=0$. At higher amplitudes, the resource deviates from the ideal coherent state superposition and thus the fidelity decreases. For comparison, we also plot the expected gate fidelity for the case of an ideal resource (the solid green/light gray line). In the experiment described below we use $\alpha=0.8$, which gives a reasonable trade-off between fidelity $(F=0.97)$, required squeezing $(V=2.6 \mathrm{~dB})$, and success probability.

The experimental setup is presented in Fig. 2. Nearly Fourier-limited picosecond pulses (4.6 ps) generated by a cavity-dumped Ti:sapphire laser with a repetition rate of $815 \mathrm{kHz}$ and a central wavelength of $830 \mathrm{~nm}$ are frequency doubled [second-harmonic generation (SHG)] by single passing a 3-mm-long periodically poled $\mathrm{KTiOPO}_{4}$ nonlinear crystal (PPKTP1). Up-converted pulses at $415 \mathrm{~nm}$ pumps a second crystal (PPKTP2) which is phase matched for degenerate collinear optical parametric amplification (OPA), thus yielding up to $3 \mathrm{~dB}$ of vacuum squeezing, in the vertical polarization. This state is used as a resource for the Hadamard gate. An adjustable fraction of a horizontally polarized mode at $830 \mathrm{~nm}$ passes the OPA crystal unchanged and serves as the input coherent state to the gate. Approximately $7.5 \%$ and

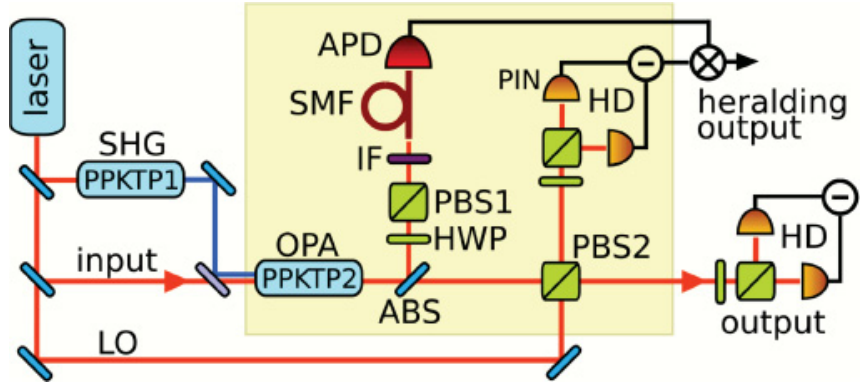

FIG. 2. (Color online) Experimental setup for the coherent state qubit Hadamard gate.

$1.5 \%$ of the coaxially propagating resource and input modes, respectively, are reflected off an asymmetric beam splitter (ABS) and transmitted through a half-wave plate (HWP) and a polarizing beam splitter (PBS1), which in combination acts as a variable beam splitter (BS), thus mixing the input mode and the resource mode. The transmittance $|t|^{2}$ of the BS is set to $25 \%$. The output is spatially and spectrally filtered by a single-mode optical fiber (SMF) and a narrow interference filter (IF) with a bandwidth of $0.05 \mathrm{~nm}$ and detected by a single-photon counting module based on a silicon avalanche photodiode (APD) with a dark count rate of $20 \pm 4$ per second. The total efficiency of the APD arm reaches $25 \pm 4 \%$.

The transmitted fraction of the modes after the asymmetric beam splitter is superimposed with a bright local oscillator (LO) at a polarizing beam splitter (PBS2). The amplitude quadrature is measured on the reflected mode by homodyne detection with a fixed relative phase set to zero. The recording of the measurement results was done by correlating the APD detection events with a synchronization signal from the laser cavity dumper through a coincidence circuit to decrease the probability of dark events. Every time a photon was detected by the APD within the accepted time slot, the homodyne signal was sampled by an oscilloscope running in a memory segmentation regime and fed to a computer where the corresponding quadrature value was processed. The state at the output of the gate is measured with another homodyne detector with the relative phase of the LO scanned over a period and then reconstructed using maximum-likelihood-based quantum state tomography [18]. In the reconstruction we corrected for the total detection efficiency of the homodyne detector, which was estimated to be $77 \pm 2 \%$, including efficiency of the photodiodes $(93 \pm 1 \%)$, visibility $(95 \pm 1 \%)$, and transmission efficiency $(93 \pm 1 \%)$.

Making a full experimental investigation of the gate performance would require access to states in the diagonal basis. In our experiment we did not have access to these diagonal states, which prevents us from performing a full characterization of the gate performance. The gate was solely tested for the computational basis states $| \pm \alpha\rangle$, which after the displacement operation $\hat{D}(\alpha)$ corresponds to the injection of $|0\rangle$ and $|2 \alpha\rangle$, where $\alpha=0.8 \pm 0.2$ in our case. The uncertainty is due to the imperfect calibration of total losses of the whole setup. As described, the gate is heralded by conditioning on two different measurement outcomes-the APD detection event and a certain outcome of the first homodyne detector. It can be seen that the conditional homodyning only plays a role 
when we inject a CSQ into the gate, i.e., when $u, v \neq 0$. With coherent states as the input, the solution is to choose a narrow heralding window that would balance the success probabilities of the gate for those basis states. For the input state $|-\alpha\rangle$ the APD detection probability was of the order of $10^{-3}$ while for the $|\alpha\rangle$ input state, the probability was of the order of $10^{-2}$. From this we can see that we need to choose a heralding window that will balance out the factor of 10 . Based on the experimental data we found its optimal position $x=0.4$ and the width of 0.02 that would give us an overall success probability of the order of $10^{-5}$.

The reconstructed output states for both input states $|-\alpha\rangle$ and $|\alpha\rangle$ can be seen in Fig. 3. For the $|-\alpha\rangle$ input, the gate yields a state which closely resembles a small odd cat state, which is what we expect from the gate operation. We found the fidelity between the prepared state and the ideal CSQ, $(|\alpha\rangle-|-\alpha\rangle) / \sqrt{N_{-}}$, is maximized for $\alpha=0.75$ and reaches a value of $F_{-\alpha}=0.65 \pm 0.04$. The nonclassicality of the superposition state produced by the Hadamard gate can be seen from the negativity of the corresponding Wigner function, which is $W(0,0)=-0.11 \pm 0.02$, which is comparable to previous experiments where photon subtraction has been used to prepare non-Gaussian states [9-15]. The nonclassical effects were also observable without correction, with a fidelity of $F_{-\alpha}=0.55 \pm 0.04$ and a value at the origin of $W(0,0)=$ $-0.05 \pm 0.02$. For the $|\alpha\rangle$ input, the output state closely resembles a squeezed state, approximating a small even CSQ, $(|\alpha\rangle+|-\alpha\rangle) / \sqrt{N_{+}}$. The fidelity between the prepared state and the ideal CSQ for $\alpha=0.75$ was found to be $F_{\alpha}=$ $0.94 \pm 0.02$.
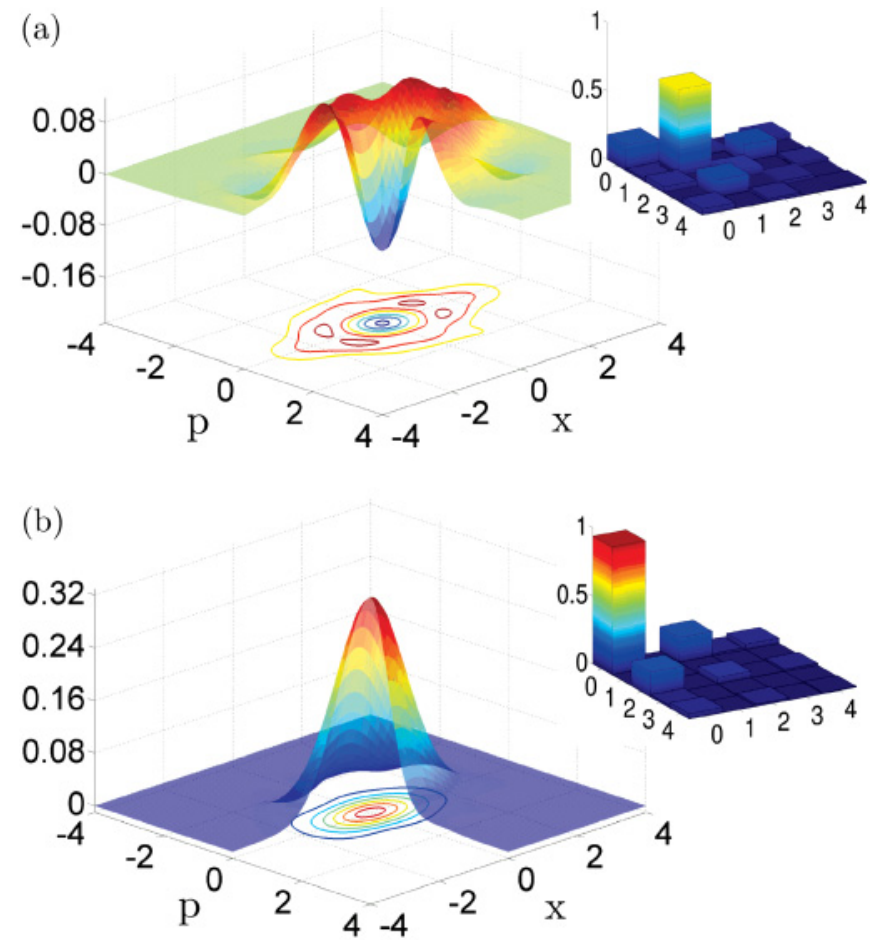

FIG. 3. (Color online) Reconstructed density matrices (insets) and calculated Wigner functions of the output states for (a) $|-\alpha\rangle$ input and (b) $|\alpha\rangle$ input.
The experimental results shown in Fig. 3 only provide a partial test of the Hadamard gate. In order to gain insight into its action on an arbitrary CSQ input, we conducted a numerical simulation of the gate, taking into account all important experimental imperfections, including realistic splitting ratios of ABS1, ABS2, and BS, losses in APD and HD channels, and the impurity of our resource squeezed state.

Our simulation starts with an arbitrary qubit in the coherent state basis $\left|\psi_{\text {in }}\right\rangle$ for which the global input state reads

$$
\hat{\rho}_{\text {in }}=\left|\psi_{\text {in }}\right\rangle_{1}\left\langle\psi_{\text {in }}|\otimes| 0\right\rangle_{2}\langle 0|\otimes| 0\rangle_{3}\langle 0| \otimes \hat{\rho}_{4}^{\mathrm{A}},
$$

where the subscripts are used to label the four participating modes and $\hat{\rho}^{\mathrm{A}}$ represents the density matrix of a squeezed thermal state used as the ancillary resource. The action of the gate can now be represented by a unitary evolution of the linear optical elements $\hat{U}$, followed by positive operator-valued measure (POVM) elements of successful heralding events $\hat{\Pi}$, with the output state given by

$$
\rho_{\text {out }}=\frac{1}{P_{\mathrm{S}}} \operatorname{Tr}_{123}\left(\hat{U} \hat{\rho}_{\text {in }} \hat{U}^{\dagger} \hat{\Pi}\right)
$$

where $P_{\mathrm{S}}=\operatorname{Tr}\left(\hat{U} \rho_{\text {in }} \hat{U}^{\dagger} \hat{\Pi}\right)$ is the success rate. $\hat{U}=$ $\hat{U}_{23}\left(t_{\mathrm{BS}}\right) \hat{U}_{12}\left(t_{\mathrm{ABS} 1}\right) \hat{U}_{34}\left(t_{\mathrm{ABS} 2}\right)$ is composed of unitary beamsplitter operations coupling the respective modes, and $\hat{\Pi}=$ $\hat{\Pi}_{1}^{\mathrm{HD}} \otimes \hat{\Pi}_{3}^{\mathrm{APD}}$ describes the inefficient homodyne and APD measurements. To parametrize a Bloch sphere of input CSQ states, we denote $u=\cos \theta$ and $v=\sin \theta \exp (\mathrm{i} \phi)$, where $\theta \in$ $[0, \pi / 2]$ and $\phi \in[0,2 \pi]$. The north and south poles correspond to the pseudo-orthogonal states $|\alpha\rangle$ and $|-\alpha\rangle$, respectively.
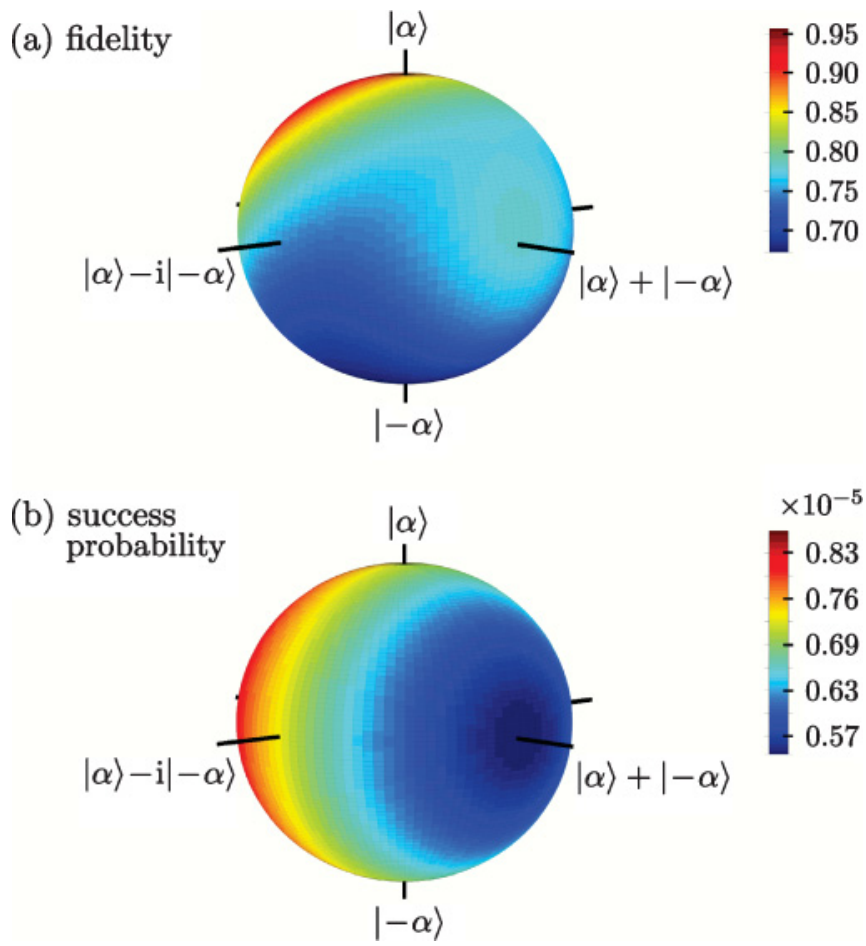

FIG. 4. (Color online) The overall quality of the gate is visualized by mapping the Bloch sphere of input CSQ onto the fidelity $F$ of the output states (a) and their corresponding success probabilities $P_{\mathrm{S}}(\mathrm{b})$. 
A mapping of this Bloch sphere onto the corresponding fidelities and success probabilities at the output is shown in Fig. 4. The fidelity spans the interval of $F \in[0.67,0.96]$ with an average value of $\bar{F}=0.78$. Particularly, for coherent states $|\alpha\rangle$ and $|-\alpha\rangle$ at the input, the fidelities of 0.88 and 0.67 are predicted, respectively, which agrees well with the actually measured values. The success probabilities associated with $|\alpha\rangle$ and $|-\alpha\rangle$ are almost equal, which confirms the correct value of the amplitude quadrature used at the HD for conditioning. The average success probability is $\bar{P}_{\mathrm{S}}=$ $7.2 \times 10^{-6}$.

Alternatively, we quantify the performance of the gate by employing the process fidelity. This quantity is based on the elegant notion that any operation can be implemented through teleportation: The desired operation is conducted onto an entangled state which is subsequently used to teleport the state on which the operation should be imparted [19]. The quality of such an operation is given by the quality of the actually transformed entangled state, which can be quantified by the fidelity with respect to the ideally transformed entangled state. We have performed a numerical simulation of the transformation of the entangled state $|\alpha, \alpha\rangle+|-\alpha,-\alpha\rangle$ and compared it to the ideally transformed state $|\alpha\rangle(|\alpha\rangle+|-\alpha\rangle) / \sqrt{N_{+}}+|-\alpha\rangle(|\alpha\rangle-|-\alpha\rangle) / \sqrt{N_{-}}$. The process fidelity resulting from this simulation reaches $\mathcal{F}=0.70$.
In conclusion, we have demonstrated a single-mode Hadamard gate for coherent state qubits on the computational basis, by using a hybrid projector consisting of a conditional homodyne detector and a photon counter. Its performance has been characterized by a set of basis states and from this we derived a model which could be used to simulate its performance for an arbitrary qubit. This implementation constitutes an important step toward the demonstration of quantum computing with macroscopic qubit states. To implement universal quantum computing, the Hadamard gate must be supplemented with a single-mode phase gate (a special case-the sign-flip gate-was recently implemented [20]) and a two-mode controlled phase gate. In addition to the implementation of these gates, another outlook is to refine the experimental techniques or propose alternate schemes that may increase the gate fidelity, and thus eventually may allow for fault-tolerant operation.

The work was financed by the Danish Research Agency (Project No. FNU 09-072623) and EU project COMPAS. P.M. acknowledges the support by Projects No. ME10156 of the Czech Ministry of Education and No. P205/10/P319 of the Czech Grant Agency. M.J. acknowledges the support by Project Nos. MSM6198959213 and LC06007 of the Czech Ministry of Education and by the Palacký University (PrF_2011_015).
[1] P. Kok et al., Rev. Mod. Phys. 79, 135 (2007).

[2] R. Raussendorf, D. E. Browne, and H. J. Briegel, Phys. Rev. A 68, 022312 (2003).

[3] E. Knill, R. Laflamme, and G. Milburn, Nature (London) 409, 46 (2001).

[4] T. C. Ralph, A. Gilchrist, G. J. Milburn, W. J. Munro, and S. Glancy, Phys. Rev. A 68, 042319 (2003).

[5] H. Jeong and T. Ralph, in Quantum Information With Continuous Variables of Atoms and Light, edited by N. J. Cerf, G. Leuchs, and E. S. Polzik (Imperial College Press, London, 2007).

[6] A. P. Lund, T. C. Ralph, and H. L. Haselgrove, Phys. Rev. Lett. 100, 030503 (2008).

[7] P. Marek and J. Fiurášek, Phys. Rev. A 82, 014304 (2010).

[8] The Hadamard transform is the unitary operation defined as $\hat{H}(x|0\rangle+y|1\rangle) \rightarrow x(|0\rangle+|1\rangle)+y(|0\rangle-|1\rangle)$, assuming $\langle 0 \mid 1\rangle=0$. In our case the coherent basis states $|\alpha\rangle$ and $|-\alpha\rangle$ are only approximately orthogonal, which opens a question of the normalization of the corresponding CSQ states. Our choice of the normalized transformations (in contrast to $| \pm \alpha\rangle \rightarrow$ $|\alpha\rangle \pm|-\alpha\rangle$ ) has the benefit of all states being physically sound and, consequently, fidelities for $\alpha \rightarrow 0$ approaching unity. On the other hand, it is true that for these small amplitudes the gate does not behave exactly as the Hadamard gate. However, this is a general problem caused by the nonorthogonality of the coherent states, and we are avoiding it by choosing reasonably large $\alpha$. Furthermore, our gate implements both the transformations (the normalized as well as the unnormalized one), switching between them just by a suitable choice of the quadrature value used at the HD for heralding of the gate.

[9] J. S. Neergaard-Nielsen et al., Phys. Rev. Lett. 105, 053602 (2010).

[10] J. Wenger, R. Tualle-Brouri, and P. Grangier, Phys. Rev. Lett. 92, 153601 (2004).

[11] J. S. Neergaard-Nielsen, B. M. Nielsen, C. Hettich, K. Molmer, and E. S. Polzik, Phys. Rev. Lett. 97, 083604 (2006).

[12] A. Ourjoumtsev et al., Science 312, 83 (2006).

[13] K. Wakui et al., Opt. Express 15, 3568 (2007).

[14] N. Namekata et al., Nat. Photonics 4, 655 (2010).

[15] T. Gerrits et al., Phys. Rev. A 82, 031802(R) (2010).

[16] S. A. Babichev, B. Brezger, and A. I. Lvovsky, Phys. Rev. Lett. 92, 047903 (2004).

[17] A. Ourjoumtsev et al., Nature (London) 448, 784 (2007).

[18] Z. Hradil, Phys. Rev. A 55, R1561 (1997); M. Ježek, J. Fiurasek, and Z. Hradil, ibid. 68, 012305 (2003); A. I. Lvovsky, J. Opt. B: Quantum Semiclassical Opt. 6, S556 (2004).

[19] D. Gottesman and I. L. Chuang, Nature (London) 402, 390 (1999); L. Slodička, M. Jezek, and J. Fiurasek, Phys. Rev. A 79, 050304(R) (2009).

[20] R. Blandino et al., e-print arXiv:1105.5510. 